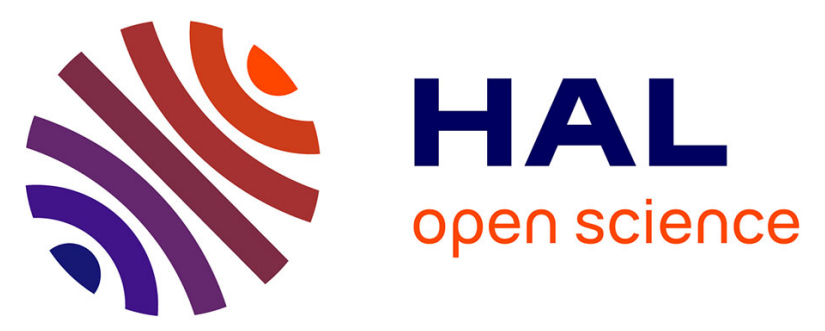

\title{
Large Surface LC-resonant Metamaterials: from Circuit Model to Modal Theory and Efficient Numerical Methods
}

\author{
Laurent Krähenbühl, Riccardo Scorretti, Arnaud Bréard, Christian Vollaire, \\ Jean Michel Guichon, Olivier Chadebec, Gérard Meunier, A \\ Urdaneta-Calzadilla, Viviane Cristine Silva, Carlos Antonio França Sartori
}

\section{To cite this version:}

Laurent Krähenbühl, Riccardo Scorretti, Arnaud Bréard, Christian Vollaire, Jean Michel Guichon, et al.. Large Surface LC-resonant Metamaterials: from Circuit Model to Modal Theory and Efficient Numerical Methods. IEEE Transactions on Magnetics, 2020, 56 (2), pp.6701504. 10.1109/TMAG.2019.2952541 . hal-02194948

\section{HAL Id: hal-02194948 \\ https://hal.science/hal-02194948}

Submitted on 6 Nov 2019

HAL is a multi-disciplinary open access archive for the deposit and dissemination of scientific research documents, whether they are published or not. The documents may come from teaching and research institutions in France or abroad, or from public or private research centers.
L'archive ouverte pluridisciplinaire HAL, est destinée au dépôt et à la diffusion de documents scientifiques de niveau recherche, publiés ou non, émanant des établissements d'enseignement et de recherche français ou étrangers, des laboratoires publics ou privés. 


\title{
Large Surface LC-resonant Metamaterials: from Circuit Model to Modal Theory and Efficient Numerical Methods
}

\author{
L. Krähenbühl ${ }^{1,2}$, R. Scorretti ${ }^{1,2}$ A. Bréard ${ }^{1,2}$, C. Vollaire ${ }^{1,2}$, Senior Member, IEEE, J.-M. Guichon ${ }^{2,3}$, O. Chadebec ${ }^{2,3}$, \\ G. Meunier ${ }^{2,3}$, A. Urdaneta-Calzadilla ${ }^{3}$, V. C. Silva ${ }^{2,4}$, C.A.F. Sartori ${ }^{2,4}$, Senior Member, IEEE \\ ${ }^{1}$ Univ Lyon, ECLyon, Ampère, CNRS, France, laurent.krahenbuhl@ec-lyon.fr - ${ }^{2}$ LIA Maxwell, CNRS (France)/CNPq (Brazil) \\ ${ }^{3}$ Univ Grenoble Alpes, Grenoble INP, G2Elab, CNRS, France $-{ }^{4}$ EP-USP, LMAG, São Paulo, Brazil
}

\begin{abstract}
We studied the harmonic magnetodynamic behavior (without free space wave propagation) of a resonant surface metamaterial, made of many identical and regularly arranged LC cells. The circuit model gives the exact solution, but it is not numerically efficient for simulating very large structures (e.g. $1000 \times 1000$ cells giving $10^{6}$ unknowns with a full $10^{6} \times 10^{6}$ matrix). For the first time, we highlight the modal characteristics of the spatial solutions, which makes it possible to explain their frequency and spatial related properties. From these results, we show under what assumptions it is possible to significantly lighten the system of equations, which opens the way to develop more efficient numerical methods.
\end{abstract}

Index Terms - LC metamaterials, resonance, circuit analysis, modal analysis, homogenization, matrix reduction, integral equations.

\section{INTRODUCTION: TYPICAL DEVICE}

$\mathrm{T}$ HE TYPICAL device that we consider was first proposed in [1], with $8 \times 8$ resonant LC cells arranged on a plane surface (Fig. 1). The goal and originality of the present work are to explain the frequency behavior of this kind of devices and to propose efficient numerical methods to simulate them even for arrangements with a far greater number, $N$, of cells (e.g. $1000 \times 1000, N=10^{6}$ ).

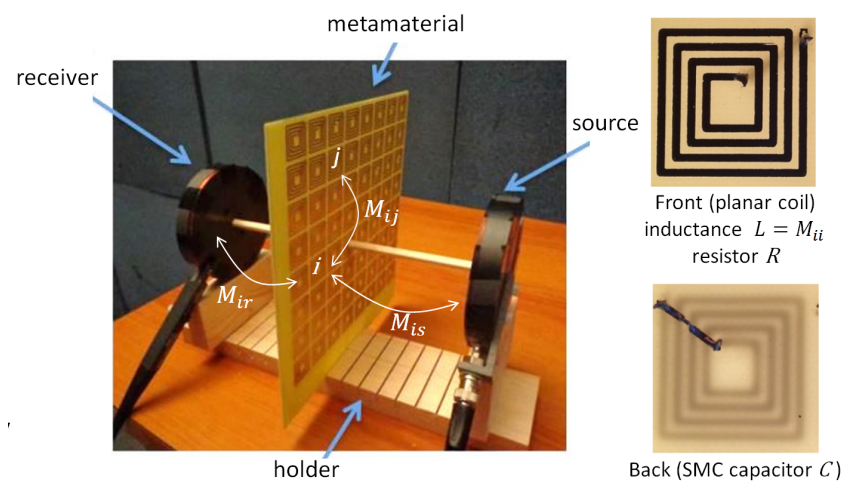

Fig. 1. Experimental setup applied to wireless power coupling studies [1]. Natural frequency of the isolated cell: $f_{0}=1 /\left(2 \pi \sqrt{M_{i i} C}\right)$.

\section{CiRCUIT ANALYSis TECHNIQUe (REFERENCE SOLUTION)}

Parasitic capacitive effects are negligible with the frequency range and geometric dimensions considered here. For quite small systems, the reference solution is then easy to obtain using the circuit analysis technique. We get a complex, full

Manuscript received Aug. 15, 2019; revised Oct. 21, 2019; accepted Nov. 6, 2019. Date of publication xxx xx, 2020; date of current version .... (Dates will be inserted by IEEE; "published" is the date the accepted

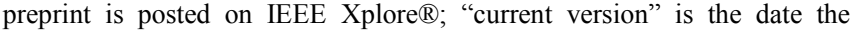
typeset version is posted on Xplore $\mathbb{R})$. Corresponding author: L. Krähenbühl (e-mail: laurent.krahenbuhl@ec-lyon.fr).

Color versions of one or more of the figures in this paper are available online at http://ieeexplore.ieee.org.

Digital Object Identifier (inserted by IEEE).
$N \times N$ matrix with main terms around the diagonal:

$\left(R+\frac{1}{\boldsymbol{j} \omega C}\right) \underline{I}_{i}+\boldsymbol{j} \omega \sum_{j=1}^{N} M_{i j} \underline{I}_{j}=-\boldsymbol{j} \omega\left(M_{i s} \underline{I}_{S}+M_{i r} \underline{I}_{r}\right)$.

The mutual inductors (matrix $M_{i j}$ and vectors $M_{i s}$ and $M_{i r}$ ) may be analytically $[2,3]$ or numerically computed. It is then possible to obtain the cell currents $I_{i}(\omega)$ and the flux in the receiver as a function of the angular frequency $\omega$. For the present preliminary approach, we suppose that the excitation current $\underline{I}_{s}$ is known, and that there is no current in the receiver $\left(\underline{I}_{r}=0\right)$. The method may be applied for other choices by considering the corresponding circuit equations.

For small structures, circuit analysis gives the exact reference solutions, but it is not practicable for large ones (a $1000 \times 1000$ cell metamaterial gives a $10^{6} \times 10^{6}$ full matrix). Moreover, it does not give insight into understanding how the device works. The modal approach, applied below for the first time in this context, provides this understanding.

\section{MODAL APPROACH}

\section{A. 1D Theory.}

To simplify the explanations, we first establish here the modal characteristics of the solutions for $2 \mathrm{D}$ field problems with 1D metamaterials, made of $N=2 n+1$ cells (Fig. 2); then the corresponding expressions for extension to a real 3D situation with 2D metamaterials will be given.

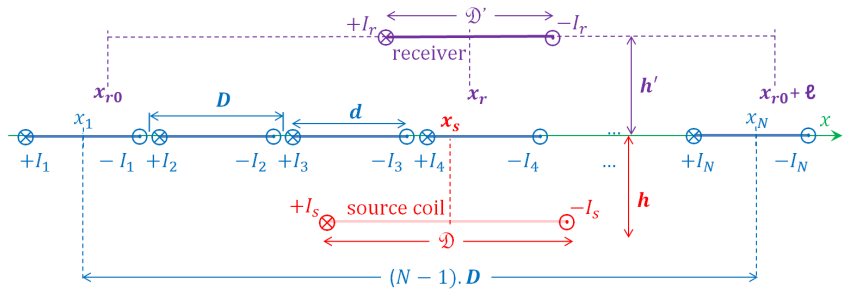

Fig. 2. 2D problem with a $1 \mathrm{D}$ linear metamaterial 


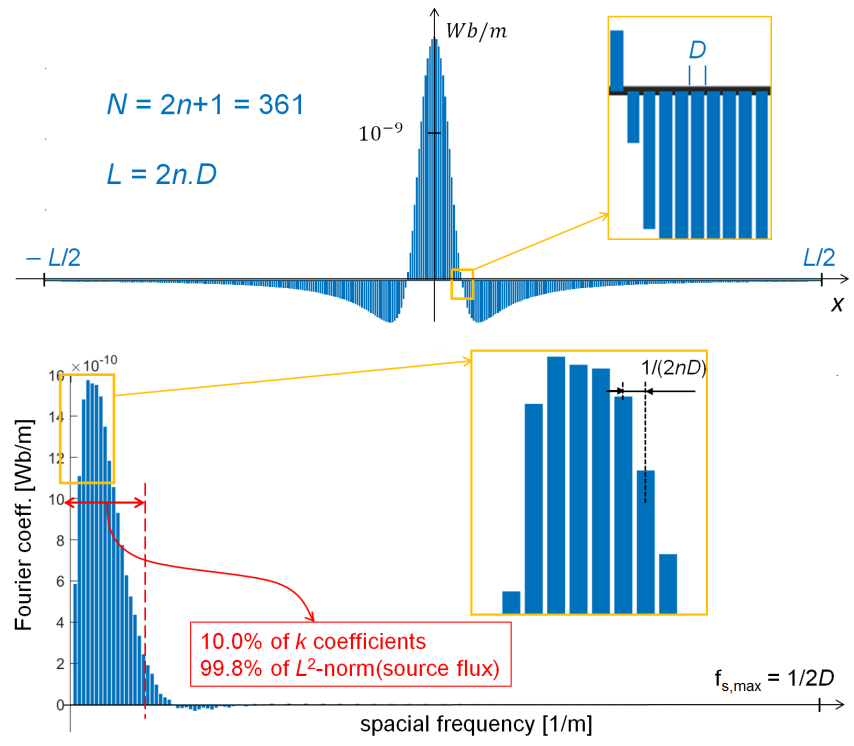

Fig. 3. Typical source flux $\phi_{i s}$ along the metamaterial (top), and its $n=181$ Fourier coefficients (bottom). With the data in Fig. 6 (size of the source coil $\gg D$ ), the 18 lowest spatial frequencies give $99.8 \%$ of the $L^{2}$-norm.

Let us develop, in spatial discrete Fourier series, the $N=2 n+1$ discrete values of the source flux, $\phi_{i s}=M_{i s} I_{s}$, (Fig.

3 ) and the solution for the current, $\underline{I}_{i}$. For the cell $i$ located at the abscissa $x_{i}$ of the metamaterial (Fig. 2), we get:

$$
\underline{\phi}_{i s}=\sum_{k=-n}^{n} \underline{\boldsymbol{\phi}}_{k s} \boldsymbol{e}^{j 2 \pi k \frac{\chi_{i}}{L}} ; \underline{I}_{i}=\sum_{k=-n}^{n} \underline{\boldsymbol{I}}_{k} \boldsymbol{e}^{\boldsymbol{j} 2 \pi k \frac{\chi_{i}}{L}}
$$

where $\underline{\phi}_{k s}$ and $\underline{\boldsymbol{I}}_{k}$ are the Fourier coefficients. Then the $\boldsymbol{j} \omega \sum_{j} M_{i j} I_{j}$ term of the circuit equation (1), which gives the flux in cell number $i$ due to all cell currents $\underline{I}_{j}(j=1, N)$, can be directly evaluated from these modal expressions by:

$$
\begin{aligned}
& \sum_{j=1}^{N} M_{i j} \underline{I}_{j}=\sum_{j=1}^{N} M_{i j} \sum_{k=-n}^{n} \underline{\boldsymbol{I}}_{k} \boldsymbol{e}^{j 2 \pi k \frac{x_{j}}{L}} \\
& =\sum_{k=-n}^{n}\left[\underline{\boldsymbol{I}}_{k} \boldsymbol{e}^{j 2 \pi k \frac{x_{i}}{L}} \sum_{j=1}^{N} M_{i j} \boldsymbol{e}^{j 2 \pi k \frac{x_{j}-x_{i}}{L}}\right] \\
& =\sum_{k=-n}^{n}\left[\underline{\mathcal{M}_{k, i}} \cdot \underline{I}_{i, k}\right]
\end{aligned}
$$

where the "modal mutual inductances", $\mathcal{M}_{k, i}$, are complex numbers, function of the mode, $k$, and cell position, $i$ :

$$
\underline{\mathcal{M}}_{k, i}=\sum_{j=1}^{N} M_{i j} e^{j 2 \pi k \frac{x_{j}-x_{i}}{L}}
$$

The mutual values, $M_{i j}$, are highly dominating for neighbor cells: as a result, $\mathcal{\mathcal { M }}_{k, i}$ is almost a real number, and a constant except near edges. Fig. 4 illustrates a 2D configuration.

The modal form of circuit equation (1), written for the $N$ modal currents, $\underline{\boldsymbol{I}}_{k}$, becomes $(i=1, N)$ :

$$
\begin{aligned}
& \sum_{k=-n}^{n}\left(R+\frac{1}{\boldsymbol{j} \omega C}+\boldsymbol{j} \omega \underline{\left.\mathcal{M}_{k, i}\right) \underline{\boldsymbol{I}_{k}} e^{j 2 \pi k} \frac{x_{i}}{L}}\right. \\
&=-\boldsymbol{j} \omega \sum_{k=-n}^{n} \underline{\boldsymbol{\phi}}_{k s} \boldsymbol{e}^{\boldsymbol{j} 2 \pi k \frac{x_{i}}{L}} .
\end{aligned}
$$

In this form, the matrix is better conditioned than in (1) but is still full. In fact, each mode, $\boldsymbol{\phi}_{k s}$, of the source field excites almost all modes of the cell currents. The edge effects and corresponding variations of $\mathcal{M}_{k, i}$ cause these mode mixtures.

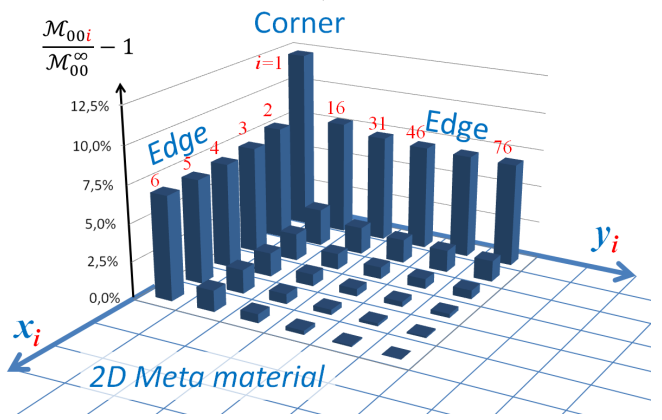

Fig. 4. Example of variations of $\mathcal{M}_{k l i}(9)$ as a function of the relative position of the cell $\left(x_{i}, y_{i}\right)$ on a 2D metamaterial of Fig. 1 type, $15 \times 15$ cells: 2D-mode $k=l=0$, given in $\%$ of the modal reference $\mathcal{M}_{00}^{\infty}(10) . \mathcal{M}_{00 i}$ are real numbers.

\section{B. $1 D$ infinite metamaterial.}

However, if we consider the theoretical case of a metamaterial without edges (geometrically infinite), the mutual mode, $k^{\prime}$, no longer depends on the position of the cell considered. Indeed, the summations in (4) turn into spatial convolutions between the mutual impedances, $M_{i j}$, and the modal functions of spatial frequencies, $f_{s}=k^{\prime} / 2 n^{\prime} D$. This leads to the new concept of "infinite modal mutual inductances", $\mathcal{M}_{k^{\prime}}^{\infty}$, which are real numbers $\left(j\right.$-evenness of $\left.M_{i j}\right)$ :

$$
\mathcal{M}_{k^{\prime}}^{\infty}=\mathcal{M}^{\infty}\left(f_{s}\right)=\lim _{m \rightarrow \infty} \sum_{j=i-m}^{i+m} M_{i j} e^{j 2 \pi f_{s}\left(x_{j}-x_{i}\right)}
$$

There is, theoretically, a number $\left(2 n^{\prime}+1\right)$ of modes that tends toward infinity, with spatial frequencies between 0 and $1 / 2 D$ (the 2 factor comes from Shannon's theorem). Numerically, it is necessary to limit the number of modes being calculated, which is equivalent to considering a repetitive structure of finite size, $L^{\prime}=2 n^{\prime} D$ (foldover distortion). We can choose, for example, $n^{\prime}=n$, taking for $L^{\prime}$ the size, $L$, of the real metamaterial.

In this case, we will find the same number of modal resonances and we have the same number of degrees of freedom, $N^{\prime}=N=2 n+1$, but the problem is not exactly the same. The numerical solution obtained with a finite number of modes corresponds, in fact, to the infinite system with antiperiodic excitation in Fig. 5.

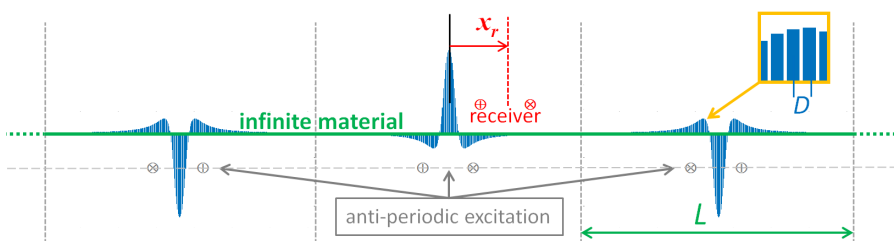

Fig 5. Infinite anti-periodic configuration corresponding to modal numerical solutions.

This modal approach will only be accurate if $L \gg D$ (size of the elementary cell), if the flux excitation is "far from the edges", and if the resulting solutions are such that the cell current in the material, $\underline{I}_{i}$, also decreases very strongly towards its edges. 
In practice, the infinite sum (6) converges very quickly and only the mutual inductances of the first 5 to 10 neighboring cells should be considered. The computation of $\mathcal{M}^{\infty}\left(f_{s}\right)$ (analytically or numerically, according to the complexity of the elementary cell configuration) is to be carried out only once for a given metamaterial because the result does not depend on the excitation. The function to be identified is generally quite regular; it is enough to calculate it for some spatial frequencies between 0 and $1 / 2 D$ and to build a polynomial interpolation. We give an example below (Fig. 6) for which a polynomial interpolation of degree 4 gives, for all modes, a relative error less than $10^{-4}$ in comparison with the numerical values of the limit (6).

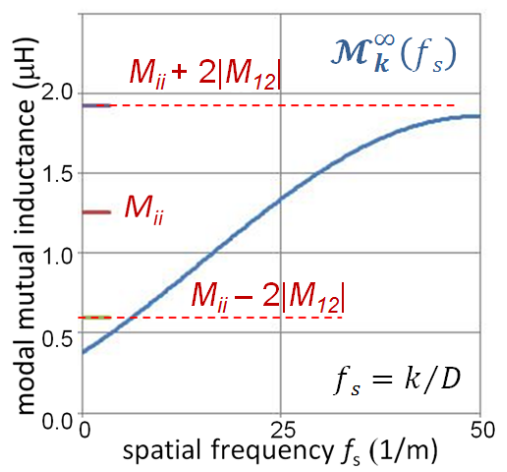

Data (see Fig. 2) for the numerical results of Fig. 3, Fig. 6, Fig. 7, and Fig. 9:

Metamaterial:

$D=10 \mathrm{~mm} ; d=9 \mathrm{~mm}$

wire $\phi=1 \mathrm{~mm}$

$N=361$

$R=1 \mathrm{~m} \Omega ; C=1.95 \mu \mathrm{F}$

Source and receiver:

$h=h^{\prime}=100 \mathrm{~mm}$

$\mathcal{D}=150 \mathrm{~mm}$

$\mathcal{D}^{\prime}=100 \mathrm{~mm}$

Depth: $1 \mathrm{~m}$

Fig. 6. Infinite modal mutual inductance $\mathcal{M}_{k}^{\infty}(6)$ as a function of spatial frequency, $f_{s}$, for the 1D metamaterial in Fig. 2.

The circuit equations (1) are then translated by decoupled modal equations ( $\mathrm{N}$ equations at 1 unknown): that is, each modal component, $k$, of the source flux, $\boldsymbol{\phi}_{k s}$, excites only the same mode, $k$, of the current:

$$
\left(R+\frac{1}{\boldsymbol{j} \omega C}+\boldsymbol{j} \omega \mathcal{M}_{k}^{\infty}\right) \underline{\boldsymbol{I}}_{k}=-\boldsymbol{j} \omega \underline{\boldsymbol{\phi}}_{k s} \quad k=-n \ldots n .
$$

The modal equation (7) highlights an electric resonance frequency, $1 /\left(2 \pi \sqrt{\mathcal{M}_{k}^{\infty} C}\right)$, for each spatial mode, $k$, the fastest spatial variations correspond to the lowest electric resonance frequencies.

The solution in the receiver's plan can also be written in modal form. This makes it possible to explain a phenomenon, initially demonstrated experimentally; the presence of the metamaterial also makes it possible to transfer energy when the receiver winding is largely off-center with respect to the source [5].

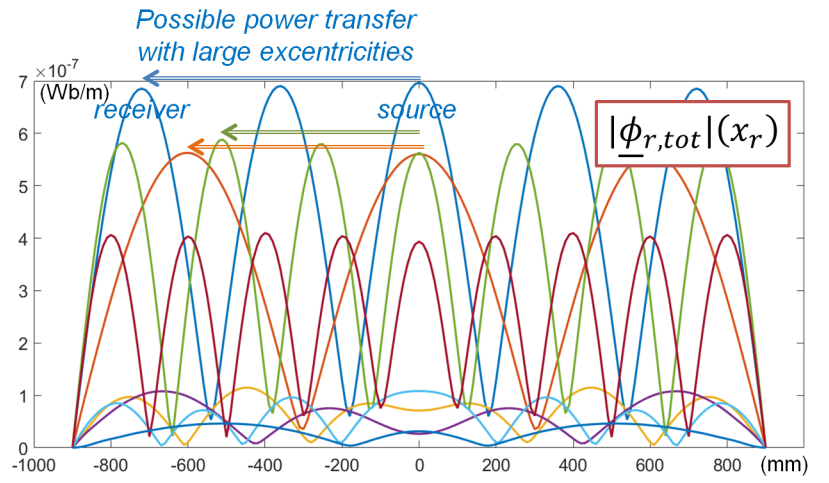

Fig. 7. Flux in the receiver of Fig 2 as a function of its $x_{r}$ position: 8 main resonant modes.
For this purpose, by a judicious choice of the source electric frequency, it is enough to excite the spatial mode in which the lobe is in front of the receiver (Fig. 7).

\section{Corresponding expressions for a 2D metamaterial.}

For a 2D metamaterial, the discrete Fourier series have 2 indexes $k$ and $l$, corresponding to both tangential directions $x$ and $y$ (Fig. 8). The main results can be preserved by adopting the necessary changes. We can then get:

$$
\begin{gathered}
\underline{\phi}_{i s}=\sum_{l=-n_{y}} \sum_{k=-n_{x}}^{n_{y}} \underline{\boldsymbol{\phi}}_{k l, s} \boldsymbol{e}^{j 2 \pi k \frac{x_{i}}{L_{x}}} \boldsymbol{e}^{j 2 \pi l \frac{y_{i}}{L_{y}},} \\
\underline{\mathcal{M}}_{k, l, i}=\sum_{j=1}^{N} M_{i j} \boldsymbol{e}^{j 2 \pi k \frac{x_{j}-x_{i}}{L_{x}}} \boldsymbol{e}^{j 2 \pi l \frac{y_{j}-y_{i}}{L_{y}}}, \\
\mathcal{M}_{k, l}^{\infty}=\sum_{j=-\infty}^{\infty} M_{i j} \boldsymbol{e}^{j 2 \pi k \frac{x_{j}-x_{i}}{L_{x}}} \boldsymbol{e}^{j 2 \pi l \frac{y_{j}-y_{i}}{L_{y}}} .
\end{gathered}
$$

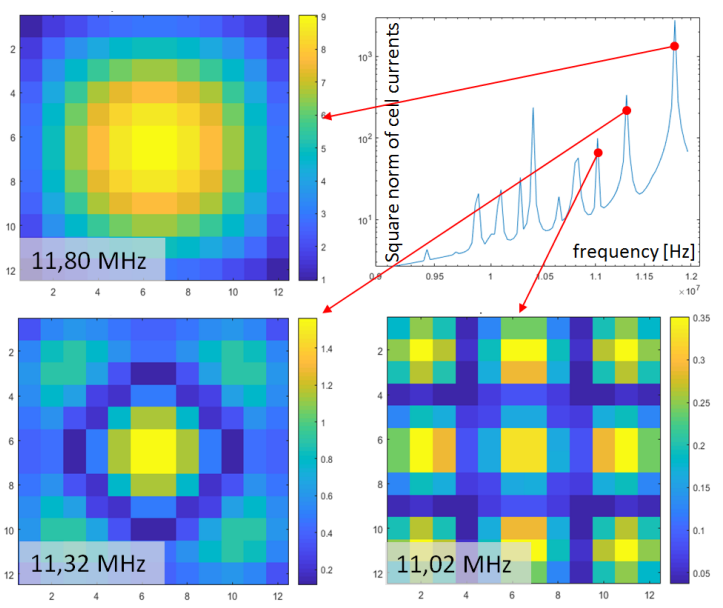

Fig. 8 . 2D resonant solutions $\left(12 \times 12\right.$ cells, $\left.f_{0}=10 \mathrm{MHz}\right)$ obtained with the complete circuit equations [4]. Colors represent the cell current modulus, $I_{i .}$.

\section{Numerical EXAMPLE (1D Metamaterial)}

In our numerical examples, we chose an excitation and a metamaterial, symmetrical with respect to $x=0$, with an odd number of cells $(N=2 n+1=361)$, and small enough for the exact solution obtained by the circuit method to be adopted as a reference solution (181 unknowns). For the modal method, symmetry leads to a priori $\underline{\boldsymbol{\phi}}_{-k s}=\underline{\boldsymbol{\phi}}_{k s} ; \underline{\boldsymbol{I}}_{-k}=\underline{\boldsymbol{I}}_{k}$, and so on.

Fig. 3 (top) shows the typical patterns of the excitation flux through the cells of the 1D metamaterial with the data given in Fig. 6, and Fig. 3 (bottom) shows its spectral decomposition as a function of the spatial frequency.

\section{A. Tests: Relevant global values and reference solution}

Using the solution obtained by the complete circuit method as a reference, we can evaluate the precision of the approximated or reduced methods.

Two global values are especially significant for the Computed Aided Design approach: First is the $L^{2}$-norm of cell currents:

$$
\left|I^{2}\right|(\omega)=\sum_{N}\left|I_{i}(\omega)\right|^{2}
$$


which expresses the intrinsic reaction of the metamaterial. Second is the ratio (12) between the flux through the receiver in the $x_{r}$ position, respectively with and without the metamaterial or its norm $\eta_{\ell}^{2}(\omega)$ on a length $\ell$, which express the frequency effect of the metamaterial for the flux transmission between the source and the receiver:

$$
\begin{gathered}
\underline{\eta}\left(x_{r}, \omega\right)=1+\sum_{j=1}^{N} M_{r j} \underline{I}_{j}(\omega) / M_{r s} \underline{I}_{s} \\
\eta_{\ell}^{2}(\omega)=\frac{1}{\ell} \int_{x_{r 0}}^{x_{r 0}+\ell}|\underline{\eta}|^{2}(x, \omega) d x .
\end{gathered}
$$

The complete reference circuit solution is obtained using the same configuration of $N=361$ cell currents $(181 \times 181$ full matrix). The global frequency behaviors (11) and (13) typically have the aspects given in Fig. 9 (blue lines).

The strong antiresonance observed for energy transmission, $\eta_{\ell}^{2}$ (13), to the receiver (Fig. 9, right) is a classic effect of the superposition of 2 shifted fields (source and reaction fields); it is not linked to the specific properties of the metamaterial. Its frequency depends on the distance, $h^{\prime}$ (Fig. 2), between the metamaterial and the receiver.

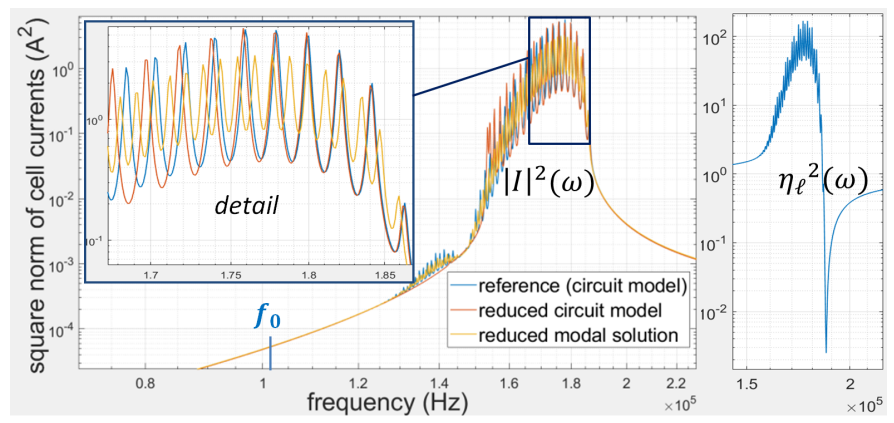

Fig. 9. Typical intrinsic (left) and transfer (right) behaviors (11) and (13).

\section{B. Modal and reduced modal solutions.}

Even if the number of equations, $n$, is of the same order of magnitude as for the circuit method, the modal method is much more efficient because their equations are independent. Moreover, we can evaluate and use only the predominant modes: if the elementary cell is small with respect to the excitation and reception coil dimensions, the amplitude of the higher modes is small and their effect on the solution will be negligible. In the typical case of Fig. 3, 10\% of the existing modes contain $99.8 \%$ of the $L^{2}$-norm of the source flux. It is then possible to reduce the number of degrees of freedom of the solution (low-pass spatial filtering) without substantially reducing its accuracy.

The global behavior of the corresponding reduced modal solution with only 37 independent equations is correctly described (see inset on Fig. 9, left, yellow line), but the resonances are not precisely obtained (peak values and frequencies), because the metamaterial is considered infinite (cf. §III. B).

\section{Reduced circuit solution}

Following the same logic, we tested a coarse first order mesh representation of cell currents, $\underline{I}_{i}$, to reduce the number of unknowns (columns of the matrix) of the exact circuit equations (1) by the same factor 10 . The shape functions of this mesh were also used to reduce the number of circuit equations (lines of the matrix) by linear combinations $(37 \times 37$ full matrix). Like the reduced modal method, this method results in a low-pass filtering of the reference solution, but this time it is possible to consider the finite dimension of the metamaterial. The accuracy is excellent for the frequency band of interest (see inset on Fig. 9, left, red line).

\section{PERSPECTIVES AND CONCLUSION}

The reduction techniques presented here for the 1D case may be easily extended for more realistic 2D metamaterials (complete tests are in progress).

The efficiency is even better because the reduction factor used (10 in the 1D case presented) applies in each of the two directions tangent to the metamaterial, reducing the number of unknowns by a factor of 100 . In the large size $2 \mathrm{D}$ case mentioned above $\left(1000 \times 1000\right.$ cells, $10^{6} \times 10^{6}$ full matrix $)$, these reduction methods lead to $10^{4}$ independent equations for the modal approximation and to a $10^{4} \times 10^{4}$ full matrix for the reduced circuit method; an ordinary laptop would be sufficient to solve it.

The most original conclusion is that, under the conditions we have explained (especially when the sizes of the source and receiver coils are larger relative to the size of the elementary cell), there are precise reduced forms of the exact equations. This result implies that a way to achieve an effective homogenization of the metamaterial should be found.

The magnetic field of the cell currents can be calculated using the integral method as the field of a distribution of normal dipoles, equivalent to the coarse mesh discretization of the cell current used for the reduced circuit method.

The homogenization step itself will consist of modifying the classical integral method to introduce (i) the close interactions (known analytically) that the integral method with coarse mesh cannot describe precisely, and (ii) the $(R+1 / \mathbf{j} \omega C)$ terms of (1).

\section{AKNOWLEDGMENT}

This work was supported by the LIA J.C. Maxwell II (CNRS LIA-817) and by the Brazilian/French project USPCOFECUB 173/18 "MaSuRe".

\section{REFERENCES}

[1] S. Nishimura, J. de Almeida, C. Vollaire, et al., "Enhancing the inductive coupling and efficiency of wireless power transmission system by using metamaterials," Momag, Curitiba, Brazil, pp.121-125, 2014. hal.archives-ouvertes.fr/hal-01064690.

[2] S. S. Mohan, et al., "Simple accurate expressions for planar spiral inductances," IEEE J. of solid-state circuits, 34-10, pp. 1419-1424, 1999

[3] F. Bilotti, et al., "Equivalent-circuit models for the design of metamaterials based on artificial magnetic inclusions," IEEE Trans. on Microwave Theory and Techniques, 55-12, pp. 2865-73, 2007

[4] Altair Flux ${ }^{\mathrm{TM}}$, Partial Element Electrical Circuit method for conductor modeling (P.E.E.C.). https://altairhyperworks.com/product/flux

[5] B. Wang, W. Yerazunis, K.H. Teo, "Wireless Power Transfer: metamaterials and array of coupled resonators," Proc. of the IEEE, 101-6, pp. 1359-1368, 2013. 